\title{
Correction: Differential transcriptional response following glucocorticoid activation in cultured blood immune cells: a novel approach to PTSD biomarker development
}

Michael S. Breen (1), Linda M. Bierer, Nikolaos P. Daskalakis (10, Heather N. Bader, louri Makotkine, Mitali Chattopadhyay, Changxin Xu, Ariela Buxbaum Grice, Anna S. Tocheva D, Janine D. Flory, Joseph D. Buxbaum, Michael J. Meaney, Kristen Brennand (iD) and Rachel Yehuda

\author{
Correction to: Translational Psychiatry \\ https://doi.org/10.1038/s41398-019-0539-x \\ published online 21 August 2019
}

This Article was originally published without the correct Supplemental Table file (Table S1 was missing). In total, there are seven Supplemental Tables, and six were in the original submission. Furthermore, Fig. 1 was misplaced in the main text; it was embedded in the manuscript file even before the results section. Both issues have now been fixed in the HTML and PDF versions of this Article.

Published online: 06 January 2020 